\title{
On the Relation between Types of Local Algebras in Different Global Representations
}

\author{
H.-J. Borchers ${ }^{1}$ and M. Wollenberg ${ }^{2}$ \\ 1 Institut für Theoretische Physik, Universität Göttingen, Bunsenstrasse 9, \\ W-3400 Göttingen, Federal Republic of Germany \\ 2 Karl-Weierstrass-Institut für Mathematik der Akademie der Wissenschaften, Mohrenstrasse 39, \\ 1086 Berlin, Federal Republic of Germany
}

Received May 15, 1990; in revised form October 10, 1990

\begin{abstract}
Let $\left\{\mathscr{A}, \mathscr{A}(O), \mathbb{R}^{d}, \alpha\right\}$ be a theory of local observables. We show that there are relations between the Connes-von Neumann types of the algebras belonging to a different global representation. For example if one representation is the vacuum representation such that the wedge algebra is of type $\mathrm{III}_{1}$ then this also is the case for other representations, provided these are connected with the vacuum by large translations.
\end{abstract}

\section{Introduction}

The type of local von Neumann algebras has been investigated in many papers (see e.g. [1, 6, 8, 9, 16]). These investigations are either based on the explicit knowledge of the algebra (free fields or generalized free fields), on the structure of lightlike translations, or on the assumption that the theory of local observables in question is connected to a Wightman field in some way or other. The latter group of results is obtained by assuming the existence of a scaling limit, which then gives rise to a free massless field whose local modular structure is known due to the analysis of Hislop and Longo [15] (for details see Fredenhagen [11], and Wollenberg [20]). In most of these investigations one finds that the Connes-von Neumann-type of the algebras under consideration is III $_{1}$.

We are interested in cases where the existence of scaling limits has not been proved, but where we have one of the following two situations: One is the case where we have an algebra and a subalgebra and in the other case we are considering two different representations. What we have in mind are cases where the two representations are connected by large translations, a situation more general than that appearing in the theory of superselection sectors initiated by Borchers [4] and developed by Doplicher, Haag, and Roberts [10] and by Buchholz and Fredenhagen [5].

We will assume that we are dealing with a $C^{*}$-algebra $\mathscr{A}$ invariant under a group $\alpha_{s}, s \in \mathbb{R}$ and that we have two states, one $\omega_{0}$ which is invariant, i.e. 
$\alpha_{s}^{\prime} \omega_{0}=\omega_{0}$ and the other which has the asymptotic property

$$
\lim _{s \rightarrow \infty} \omega\left(A \alpha_{s}(B) C\right)=\omega(A C) \omega_{0}(\beta),
$$

which means it is asymptotically connected with $\omega_{0}$.

In Sects. II and III we formulate and prove some general results and these will be applied to the theory of local observables in the last section.

\section{The Connes Spectrum and Large Translations}

Since in the situations appearing in physics one cannot exclude that the von Neumann algebras in question have a center we will deal always with the invariant

$$
S^{\prime}(\mathscr{M})=\cap \operatorname{spec} \Delta_{\omega},
$$

where $\omega$ runs over all normal states of $\mathscr{M}$, and $\operatorname{spec} \Delta_{\omega}$ denotes the spectrum of the modular operator $\Delta_{\omega}$ associated with $\omega . S^{\prime}(\mathscr{M})$ is an algebraic invariant and generalizes Connes invariant $S(\mathscr{M})$ which was defined when $\mathscr{M}$ is a factor [7]. We recall that $\Delta_{\omega}$ denotes the modular operator of the pair $\left\{\omega, \mathscr{M}_{E}\right.$ with $\mathscr{M}_{E}=E \mathscr{M} E$ and $E$ the smallest projection in $\mathscr{M}$ with $\left.\omega(E)=1\right\}$.

If $F$ is a projection in the center of $\mathscr{M}$ then one has

$$
S^{\prime}(\mathscr{M}) \subset S^{\prime}(F \mathscr{M}) \text {. }
$$

If in particular $S^{\prime}(\mathscr{M})=\mathbb{R}^{+}$as it appears mostly in the theory of local observables then this implies that in the central decomposition of $\mathscr{M}$ appear only factors of the Connes-von Neumann type III $_{1}$.

We want to investigate the situation described as follows:

1) Let $\{\mathscr{A}, \mathbb{R}, \alpha\}$ be a $C^{*}$-dynamical system, i.e. $\mathscr{A}$ is a $C^{*}$-algebra and $\alpha: \mathbb{R} \rightarrow$ Aut $\mathscr{A}$ is a representation of $\mathbb{R}$ by automorphisms of $\mathscr{A}$.

2) Let $\mathscr{A} \supset \mathscr{B} \supset \mathscr{C}$ be sub- $C^{*}$-algebras subject to the additional conditions:

(1) $\mathscr{B}$ is $\alpha_{s}$ invariant, i.e. $\alpha_{s} \mathscr{B}=\mathscr{B}$ for all $s \in \mathbb{R}$.

(2) $\alpha_{s} \mathscr{C} \subset \mathscr{C}$ for all $s \in \mathbb{R}^{+}$, i.e. $\left\{\alpha_{s} ; s \in \mathbb{R}^{+}\right\}$defines a semigroup of morphisms of $\mathscr{C}$.

Next we introduce the following

II.1. Notation. Let $\{\mathscr{A}, \mathbb{R}, \alpha\}$ be a $C^{*}$-dynamical system and $\mathscr{B} \subset \mathscr{A}$ such that

$$
\alpha_{s} \mathscr{B} \subset \mathscr{B} \text { for all } s \in \mathbb{R}^{+}
$$

then we say $\mathscr{B}$ is $\mathscr{A}$ absorbing if there exists a norm dense family $\mathscr{F} \subset \mathscr{A}$ such that for every $F \in \mathscr{F}$ exist $s(F) \in \mathbb{R}^{+}$with

$$
\alpha_{t} F \in \mathscr{B} \text { for } t>s(F) \text {. }
$$

This family $\mathscr{F}$ can obviously be chosen to be ${ }^{*}$-invariant.

3) In the following we will assume that the algebra $\mathscr{C}$ absorbs the algebra $\mathscr{B}$ in the sense of Definition II.1.

We need another notation, which is representation dependent.

II.2 Notation. Let $\mathscr{A}$ be a $C^{*}$-algebra and $\mathscr{B} \subset \mathscr{A}$ a sub-C $C^{*}$-algebra. Let $\pi$ be a representation of $\mathscr{A}$, then we say $\mathscr{B}$ fulfills the quasi-duality condition with respect to $\pi$ iff with

$$
\mathscr{B}^{c}=\{X \in \mathscr{A} ;[X, B]=0, \forall B \in \mathscr{B}\}
$$


one has

$$
\pi\left(\mathscr{B}^{c}\right)^{\prime \prime}=\pi(\mathscr{B})^{\prime} .
$$

Remark. (i) If $\mathscr{B} \subset \mathscr{A}$ and if $\mathscr{B}$ fulfills the quasi-duality condition then the von Neumann algebra generated by $\pi\left(\mathscr{B}^{c}\right) \cup \pi(\mathscr{B})$ is of type one and consequently this is true also for $\pi(\mathscr{A})$. But the centers of $\left\{\pi\left(\mathscr{B}^{c}\right) \cup \pi(\mathscr{B})\right\}^{\prime \prime}$ and of $\pi(\mathscr{A})$ are not necessarily the same.

(ii) We have used the name quasi-duality for the property described in II.2 because the name duality has a definite meaning in the theory of local observables. The property we are using here is similar to usual duality assumption but not connected to any geometrical structure.

(iii) If we are dealing with a theory of local observables which is invariant under Poincare- and C.P.T.-transformations then the quasi-duality holds in the vacuum sector (see e.g. [3]).

4) If $\omega_{0} \in \mathscr{A}^{*}$ is an $\alpha_{s}$ invariant state and $\left\{\pi_{0}, \mathscr{H}, \Omega_{0}\right\}$ its G.N.S. representation then we assume that $\pi_{0}(\mathscr{A})$ is weakly clustering meaning we assume for $A, B$, $C \in \mathscr{A}$

$$
\lim _{s \rightarrow \infty} \omega_{0}\left(B \alpha_{s}(A) C\right)=\omega_{0}(B C) \omega_{0}(A) .
$$

5) The representation $\left\{\pi_{0}, \mathscr{H}, \Omega_{0}\right\}$ induced by $\omega_{0}$ shall be such that $\Omega_{0}$ is cyclic and separating for $\pi_{0}(\mathscr{B})$. Moreover, we assume that $\pi_{0}(\mathscr{B})$ fulfills the quasiduality condition. Next we want to generalize the situation described before to the case where one has two states.

II.3. Notation. Let $\{\mathscr{A}, \mathbb{R}, \alpha\}$ be a $C^{*}$-dynamical system. Let $\omega_{0}$ and $\omega$ be two states of $\mathscr{A}$. We say $\omega$ is asymptotically connected with $\omega_{0}$ if for arbitrary $A, B$, $C \in \mathscr{A}$ the relation

$$
\lim _{s \rightarrow \infty} \omega\left(A \alpha_{s}(B) C\right)=\omega(A C) \omega_{0}(B)
$$

holds.

Note that the definition of asymptotically connectedness implies that $\omega_{0}$ is an $\alpha_{s}$ invariant state.

With these notations one obtains:

II.4. Lemma. Let $\{\mathscr{A}, \mathbb{R}, \alpha\}$ be a $C^{*}$-dynamical system and $\omega, \omega_{0}$ be two states of $\mathscr{A}$. Denote by $\{\pi, \mathscr{H}, \Omega\}$ the G.N.S. representation of $\omega$. Assume that $\omega$ is asymptotically connected with $\omega_{0}$. Then every $\pi$ normal state is asymptotically connected with $\omega_{0}$.

Proof. Let $\psi \in \mathscr{H}$ be a unit vector. Assume $A, B, C \in \mathscr{A}$ and give $\varepsilon>0$. By construction of $\mathscr{H}$ we can find $D \in \pi(\mathscr{A})$ with $\|\psi-D \Omega\| \leq \frac{\varepsilon}{5\|A\|\|B\|\|C\|}$
Furthermore there exists $s_{0}$ such that

$$
\left|\left(\Omega, D^{*} \pi\left(A \alpha_{S}(B) C\right) D \Omega\right)-\left(\Omega, D^{*} \pi(A C) D \Omega\right) \omega_{0}(B)\right|<\frac{\varepsilon}{5}
$$

for $s>s_{0}$. With these estimates we obtain for $s>s_{0}$,

$$
\begin{aligned}
\mid(\psi, \pi & \left.\left(A \alpha_{s}(B) C\right) \psi\right)-(\psi, \pi(A C) \psi) \omega_{0}(B) \mid \\
\quad \leq & \left|\left(\{\psi-D \Omega\}, \pi\left(A \alpha_{s}(B) C\right) \psi\right)\right|+\left|(\{\psi-D \Omega\}, \pi(A C) \psi) \omega_{0}(B)\right| \\
& +\left|\left(D \Omega, \pi\left(A \alpha_{s}(B) C\right)\{\psi-D \Omega\}\right)\right|+\left|(D \Omega, \pi(A C)\{\psi-D \Omega\}) \omega_{0}(B)\right| \\
& +\left|\left(\Omega, D^{*} \pi\left(A \alpha_{s}(B) C\right) D \Omega\right)-\left(\Omega, D^{*} \pi(A C) D \Omega\right) \omega_{0}(B)\right| \leq \varepsilon .
\end{aligned}
$$


Since $\varepsilon$ is arbitrary we have that $(\psi, \cdot \psi)$ is asymptotically connected with $\omega_{0}$. Using that every $\pi$ normal state can be presented by a norm converging sum of $\pi$ vector states we get the same result for $\pi$ normal states.

After this preparation we get the following result.

II.5. Theorem. Let $\{\mathscr{A}, \mathbb{R}, \alpha\}$ be a $C^{*}$-dynamical system and let $\mathscr{C} \subset \mathscr{B} \subset \mathscr{A}$ be sub-C $C^{*}$-algebras, with $\mathscr{B}$ is $\alpha_{s}$ invariant, $\alpha_{s} \mathscr{C} \subset \mathscr{C}$ for $s \in \mathbb{R}^{+}$, and $\mathscr{C}$ absorbing for $\mathscr{B}$. Let $\omega, \omega_{0}$ be two states on $\mathscr{A}$ such that $\Omega_{0}$ is cyclic and separating for $\pi_{0}(\mathscr{B})$, and $\pi_{0}(\mathscr{B})$ fulfills the quasi-duality condition. Assume moreover that $\omega$ is asymptotically connected with $\omega_{0}$. Then one has:

$$
S^{\prime}\left(\pi_{0}(\mathscr{B})^{\prime \prime}\right) \subseteq \operatorname{spec} \Delta_{\Omega_{0}}^{\pi_{0}(\mathscr{B})^{\prime \prime}} \subseteq S^{\prime}\left(\pi(\mathscr{C})^{\prime \prime}\right) .
$$

The inclusion on the left-hand side is an equality if $\omega_{0}$ is $\alpha_{s}$ asymptotic abelian.

Proof. By assumption we know that the vector $\Omega_{0}$ is cyclic and separating for $\pi_{0}(\mathscr{B})^{\prime \prime}$. Moreover, the state $\omega_{0}$ is $\alpha_{s}$ invariant. If the system $\left\{\mathscr{B}, \alpha_{s}, \omega_{0}\right\}$ is weakly clustering, then by a result of Araki [2] and Størmer [19] the set $S^{\prime}\left(\pi_{0}(\mathscr{B})^{\prime \prime}\right)$ coincides with $\operatorname{spec} \Delta_{\Omega_{0}}$ (for the algebra $\left.\pi_{0}(\mathscr{B})^{\prime \prime}\right)$. For showing the inclusion on the right-hand side, we use Fredenhagen's generalization [11] of the criterion of Størmer [19]. But we remark first that this condition holds also for the eigenvalue zero, because then Fredenhagen's condition coincides with that of Størmer. Let $\lambda \in \operatorname{spec} \Delta_{\Omega_{0}}$ and $\varphi$ be a $\pi$ normal state then we have to find for any $\varepsilon>0$ an operator $A_{\varepsilon} \in \pi(\mathscr{C})^{\prime \prime}$ such that

$$
\varphi\left(A_{\varepsilon}^{*} A_{\varepsilon}\right) \geq 1
$$

and

$$
\left|\varphi\left(A_{\varepsilon} B\right)-\lambda \varphi\left(B A_{\varepsilon}\right)\right| \leq \varepsilon\left\{\varphi\left(B^{*} B\right)+\lambda \varphi\left(B B^{*}\right)\right\}^{1 / 2}
$$

holds for every $B \in \pi(\mathscr{C})^{\prime \prime}$. By the choice of $\lambda$ exists an operator $A_{1} \in \pi_{0}(\mathscr{B})^{\prime \prime}$ with

$$
\left\|A_{1} \Omega_{0}\right\| \geq 1+\frac{\varepsilon}{2}
$$

and

$$
\left\|\left(\Delta^{1 / 2}-\lambda^{1 / 2}\right) A_{1} \Omega_{0}\right\| \leq \frac{\varepsilon}{8} .
$$

$\Delta, J$ is the modular operator respectively modular conjugation of the pair $\left\{\pi_{0}(\mathscr{B})^{\prime \prime}, \Omega_{0}\right\}$. Using the notation $C_{1}=J A_{1}^{*} J$ and the invariance of $\Omega_{0}$ under $J$ we can replace (4) by

$$
\|\left(C_{1}-\lambda^{1 / 2} A_{1}\right) \Omega_{0} \mid \leq \frac{\varepsilon}{8} .
$$

This equation gives also

$$
\begin{aligned}
\left\|\left(C_{1}-\lambda^{1 / 2} A_{1}\right) \Omega_{0}\right\| & =\left\|J C_{1} \Omega-\lambda^{1 / 2} J A_{1} \Omega_{0}\right\| \\
& =\left\|A_{1}^{*} \Omega_{0}-\lambda^{1 / 2} S^{*} J S A_{1} \Omega_{0}\right\|=\left\|A_{1}^{*} \Omega-\lambda^{1 / 2} S^{*} J A_{1}^{*} \Omega\right\| \\
& =\left\|A_{1}^{*} \Omega_{0}-\lambda^{1 / 2} S^{*} C_{1} \Omega_{0}\right\|,
\end{aligned}
$$

and consequently

$$
\left\|\left(A_{1}^{*}-\lambda^{1 / 2} C_{1}^{*}\right) \Omega_{0}\right\| \leq \frac{\varepsilon}{8}
$$


Next we use that $\pi_{0}(\mathscr{B})$ is dense in $\pi_{0}(\mathscr{B})^{\prime \prime}$ in the strong- ${ }^{*}$-topology. Hence exists $A_{2} \in \pi_{0}(\mathscr{B})$ with

$$
\left\|\left(A_{2}-A_{1}\right) \Omega_{0}\right\|+\left\|\left(A_{2}^{*}-A_{1}^{*}\right) \Omega_{0}\right\| \leq \frac{\varepsilon}{8} .
$$

By quasi-duality $\pi_{0}\left(\mathscr{B}^{c}\right)$ is strong- ${ }^{*}$-dense in $\pi_{0}\left(\mathscr{B}^{\prime}\right)$. Hence there is $C_{2} \in \mathscr{B}^{c}$ with

$$
\left\|\left(\pi_{0}\left(C_{2}\right)-C_{1}\right) \Omega_{0}\right\|+\left\|\left(\pi_{0}\left(C_{2}^{*}\right)-C_{1}^{*}\right) \Omega_{0}\right\| \leq \frac{\varepsilon}{8} .
$$

Now we are making use of the fact that the algebra $\mathscr{C}$ is absorbing for $\mathscr{B}$. This means there exists a norm-dense and ${ }^{*}$-invariant subfamily $\mathscr{F} \subset \mathscr{B}$ which is absorbed by $\mathscr{C}$ by means of large translations. So we find $A_{3} \in \mathscr{F}$ with

$$
\left\|\pi_{0}\left(A_{3}\right)-A_{2}\right\| \frac{\varepsilon}{8}
$$

Let now $s_{0}$ be such that

$$
\alpha_{s} A_{3} \in \mathscr{C} \text { for } s>s_{0}
$$

and $s_{1}$ be such that

$$
\begin{aligned}
& \left|\varphi\left(\pi\left(\alpha_{s}\left\{\left(\lambda^{1 / 2} A_{3}^{*}-C_{2}^{*}\right)\left(\lambda^{1 / 2} A_{3}-C_{2}\right)\right\}\right)\right)-\omega_{0}\left(\left(\lambda^{1 / 2} A_{3}^{*}-C_{2}^{*}\right)\left(\lambda^{1 / 2} A_{3}-C_{2}\right)\right)\right| \\
& \quad+\mid \varphi\left(\pi\left(\alpha_{s}\left\{\left(A_{3}-\lambda^{1 / 2} C_{2}\right)\left(A_{3}^{*}-\lambda^{1 / 2} C_{2}^{*}\right)\right\}\right)\right) \\
& \quad-\omega_{0}\left(\left(A_{3}-\lambda^{1 / 2} C_{2}\right)\left(A_{3}^{*}-\lambda^{1 / 2} C_{2}^{*}\right)\right) \mid \leq \frac{\varepsilon^{2}}{4} \quad \text { for } \quad s>s_{1},
\end{aligned}
$$

and let $s_{2}$ be such that

$$
\left|\varphi\left(\pi\left(\alpha_{s}\left(A_{3}^{*} A_{3}\right)\right)\right)-\omega_{0}\left(A_{3}^{*} A_{3}\right)\right| \leq \frac{\varepsilon}{2} \quad \text { for } \quad s>s_{2} .
$$

Assume now $s>\max \left\{s_{0}, s_{1}, s_{2}\right\}$ then one has $\alpha_{s} A_{3} \in \mathscr{C}$ and one finds by using Eqs. (3), (5), (7), and (10):

$$
\varphi\left(\pi\left(\alpha_{S} A_{3}^{*} A_{3}\right)\right)^{1 / 2} \geq\left\{\omega_{0}\left(A_{3}^{*} A_{3}\right)-\left|\varphi\left(\pi\left(\alpha_{S} A_{3}^{*} A_{3}\right)\right)-\omega_{0}\left(A_{3}^{*} A_{3}\right)\right|\right\}^{1 / 2} \geq 1 .
$$

This shows $\pi\left(\alpha_{2} A_{3}\right)$ fulfills condition (1). Furthermore one estimates by using the inequalities $\left(4^{\prime}\right),\left(4^{\prime \prime}\right),(5),(6),(7)$, and (9):

$$
\begin{aligned}
\mid \varphi( & \left.\left(\alpha_{s}\left(A_{3}\right)\right) B\right)-\lambda \varphi\left(B \pi\left(\alpha_{s} A_{3}\right)\right) \mid \\
\leq & \varphi\left(B^{*} B\right)^{1 / 2} \varphi\left(\pi\left(\alpha_{s}\left\{\left(A_{3}-\lambda^{1 / 2} C_{2}\right)\left(A_{3}^{*}-\lambda^{1 / 2} C_{2}^{*}\right)\right\}\right)\right)^{1 / 2} \\
& +\lambda^{1 / 2} \varphi\left(B B^{*}\right)^{1 / 2} \varphi\left(\pi\left(\alpha_{s}\left\{\left(\lambda^{1 / 2} A_{3}^{*}-C_{2}^{*}\right)\left(\lambda^{1 / 2} A_{3}-C_{2}\right)\right\}\right)\right)^{1 / 2} \\
\leq & \varphi\left(B^{*} B\right)^{1 / 2}\left\{\omega_{0}\left(\left\{A_{3}-\lambda^{1 / 2} C_{2}\right\}\left\{A_{3}^{*}-\lambda^{1 / 2} C_{2}^{*}\right\}\right)+\frac{\varepsilon^{2}}{4}\right\}^{1 / 2} \\
& +\lambda^{1 / 2} \varphi\left(B B^{*}\right)^{1 / 2}\left\{\omega_{0}\left(\left\{\lambda^{1 / 2} A_{3}^{*}-C_{2}^{*}\right\}\left\{\lambda^{1 / 2} A_{3}-C_{2}\right\}\right)+\frac{\varepsilon^{2}}{4}\right\}^{1 / 2} \\
\leq & \varepsilon\left\{\varphi\left(B^{*} B\right)+\lambda \varphi\left(B B^{*}\right)\right\}^{1 / 2} .
\end{aligned}
$$

Hence $\pi\left(\alpha_{s} A_{3}\right)$ fulfills also condition (2) and the theorem is proved.

Remark. The results obtained in Theorem II.5 can be generalized in several directions. 
(i) The limit requirements in 4) and II.3 can be weakend; for instance it can be replaced by

$$
\lim _{T \rightarrow \infty} \frac{1}{T} \int_{T}^{2 T} \omega\left(A \alpha_{s}(B) C\right) d s=\omega(A C) \omega_{0}(B)
$$

or by any other averaging procedure.

(ii) It is not necessary that the group in question is isomorphic to $\mathbb{R}$. Any other group carrying an order structure will do the same job.

(iii) It is even not important to have a group. Any directed system $\left\{\alpha_{j}\right\} \subset$ Aut $\mathscr{A}$ will be sufficient provided the requirements 1)-5) are formulated correspondingly.

\section{On the Converse Problem}

In the last section we have discussed the situation where one can conclude that the Connes-invariant of the modular operators is contained in that of some subalgebra. Usually one is interested in proving the equality of these sets. The converse inclusion, however, is not easily obtained. The only case we can deal with is the equality of the spectrum of modular operators belonging to the same vector, but to different von Neumann algebras.

III.1 Theorem. Assume $\mathscr{M} \subset \mathscr{N}$ are von Neumann algebras acting on the same Hilbert space $\mathscr{N}$. Assume the unitvector $\Omega \in \mathscr{H}$ is cyclic for $\mathscr{M}$ then

$$
\operatorname{spec} \Delta_{\Omega}^{\mathcal{N}} \supseteq \operatorname{spec} \Delta_{\Omega}^{\mathscr{M}} .
$$

Proof. Assume $\lambda \in \operatorname{spec} \Delta_{\Omega}^{\mathscr{M}}$ and $\lambda \neq 0$, and let $E$ be the maximal projection in $\mathscr{M}$ with $\omega(E)=1$, then to every $\varepsilon>0$ we can find $A \in \mathscr{M}_{E}$ with $\|A \Omega\| \geq 1$ and

$$
|(\Omega, A B \Omega)-\lambda(\Omega, B A \Omega)| \leq \varepsilon\left\{\left(\Omega, B^{*} B \Omega\right)+\lambda\left(\Omega, B B^{*} \Omega\right)\right\}^{1 / 2} .
$$

Let $J$ be the modular conjugation of $\{\mathscr{N}, \Omega\}$. Then one finds by using $J B J \in \mathscr{N}^{\prime}$,

$$
\begin{aligned}
& \left|\left(\left\{J B+\lambda^{1 / 2} B^{*}\right\} \Omega,\left\{J A^{*}-\lambda^{1 / 2} A\right\} \Omega\right)\right| \\
& \quad=|(\Omega, A B \Omega)-\lambda(\Omega, B A \Omega)| \leq \varepsilon\left\{\|B \Omega\|^{2}+\left(\lambda^{1 / 2}\left\|B^{*} \Omega\right\|\right)^{2}\right\}^{1 / 2} \\
& \quad=\varepsilon\left\{\|J B \Omega\|^{2}+\left(\lambda^{1 / 2}\left\|B^{*} \Omega\right\|\right)^{2}\right\}^{1 / 2}
\end{aligned}
$$

We have $J B \Omega=J S_{\mathscr{N}} B^{*} \Omega=\Delta_{\mathcal{N}}^{1 / 2} B^{*} \Omega$. Writing $\Delta$ instead of $\Delta_{\mathcal{N}}$ and using that $\Delta^{1 / 2}$ is non-negative one has

$$
\left\{\|J B \Omega\|^{2}+\left(\lambda^{1 / 2}\left\|B^{*} \Omega\right\|\right)^{2}\right\}^{1 / 2} \leq\left\|\left(\Delta^{1 / 2}-\lambda^{1 / 2}\right) B^{*} \Omega\right\| .
$$

This gives us

$$
\mid\left(\left\{\Delta^{1 / 2}+\lambda^{1 / 2}\right\} B^{*} \Omega,\left\{J A^{*}-\lambda^{1 / 2} A\right\} \Omega\right) \leq \varepsilon\left\|\left(\Delta^{1 / 2}+\lambda^{1 / 2}\right) B^{*} \Omega\right\| .
$$

Since $\mathscr{M} \Omega$ is dense in $\mathscr{H}$ and since $-\lambda^{1 / 2}$ does not belong to the spectrum of $\Delta^{1 / 2}$ also $\left(\Delta^{1 / 2}+\lambda^{1 / 2}\right) \mathscr{M} \Omega$ is dense in $\mathscr{H}$. From this we conclude

$$
\left\|\left(J A^{*}-\lambda^{1 / 2} A\right) \Omega\right\|=\left\|\left(\Delta^{1 / 2}-\lambda^{1 / 2}\right) A \Omega\right\| \leq \varepsilon,
$$

and hence $\lambda^{1 / 2}$ is an eigenvalue of $\Delta^{1 / 2}=\Delta_{\mathscr{N}}^{1 / 2}$ since $\|A \Omega\| \geq 1$ by construction. If $0=\lambda \in \operatorname{spec} \Delta_{\Omega}^{\mathscr{M}}$ then Størmers condition tells us there exists for every $\varepsilon>0$ 
an operator $A \in \mathscr{M}_{E}$ with $\|A \Omega\| \geq 1$ and $\left\|A^{*} \Omega\right\|<\varepsilon$. But since $A \in \mathcal{N}$ one has also

$$
\left\|S_{\mathscr{N}} A \Omega\right\|=\left\|\Delta_{\mathscr{N}}^{1 / 2} A \Omega\right\|<\varepsilon
$$

and therefore 0 belongs also to the spectrum of $\Delta_{\mathscr{N}}^{1 / 2}$. In both cases we made use of the fact, if $E^{\prime}$ is the maximal projection in $\mathscr{N}^{\prime}$ with $\omega\left(E^{\prime}\right)=1$, then $E^{\prime} \geq E$ and hence $\mathscr{M}_{E} \subset \mathscr{N}_{E^{\prime}}$.

Next we want to combine this result with that of the last section.

III.2 Theorem. Let $\{\mathscr{A}, \mathbb{R}, \alpha\}$ be a $C^{*}$-dynamical system and let $\mathscr{C} \subset \mathscr{B} \subset \mathscr{A}$ be sub-C $C^{*}$-algebras, $\mathscr{B}$ invariant, $\alpha_{s} \mathscr{C} \subset \mathscr{C}$ for $s \in \mathbb{R}^{+}$and $\mathscr{C}$ absorbing for $\mathscr{B}$. Let $\omega_{0}$ be an $\alpha_{s}$ invariant state and $\left\{\pi_{0}, \mathscr{H}, \Omega_{0}\right\}$ its G.N.S. representation. Let $\pi_{0}(\mathscr{A})$ be weakly $\alpha_{s}$ asymptotically abelian, $\pi_{0}(\mathscr{B})$ fulfill the quasi-duality condition, and let $\Omega_{0}$ is cyclic and separating for $\pi_{0}(\mathscr{B})$ and $\pi_{0}(\mathscr{C})$. Then one has

$$
S^{\prime}\left(\pi_{0}(\mathscr{B})^{\prime \prime}\right)=S^{\prime}\left(\pi_{0}(\mathscr{C})^{\prime \prime}\right) .
$$

Proof. The conditions of Theorem II.5 are fulfilled, if we choose $\omega=\omega_{0}$. Therefore we get $S^{\prime}\left(\pi_{0}(\mathscr{B})^{\prime \prime}\right) \subset S^{\prime}\left(\pi_{0}(\mathscr{C})^{\prime \prime}\right)$. On the other hand one has

$$
S^{\prime}\left(\pi_{0}(\mathscr{C})^{\prime \prime}\right) \subseteq \operatorname{spec} \Delta_{\Omega_{0}}^{\pi_{0}(\mathscr{C})^{\prime \prime}}
$$

and by Theorem III.1

$$
\operatorname{spec} \Delta_{\Omega_{0}}^{\pi_{0}(\mathscr{G})^{\prime \prime}} \subseteq \operatorname{spec} \Delta_{\Omega_{0}}^{\pi_{0}(\mathscr{B})^{\prime \prime}}
$$

Since $\pi_{0}(\mathscr{B})$ is asymptotically abelian and $\Omega_{0}$ is invariant one has

$$
\operatorname{spec} \Delta_{\Omega_{0}}^{\pi_{0}(\mathscr{B})^{\prime \prime}}=S^{\prime}\left(\pi_{0}(\mathscr{B})^{\prime \prime}\right) .
$$

This together implies

$$
S^{\prime}\left(\pi_{0}(\mathscr{B})^{\prime \prime}\right)=S^{\prime}\left(\pi_{0}(\mathscr{C})^{\prime \prime}\right)
$$

\section{Applications}

Our next task is to apply the results of the last two sections to situations of physical interest. With reference to the assumptions of Theorem II.5 we need asymptotic abelian systems. These appear in statistical mechanics as well as in quantum field theory. In quantum field theory we use space-translations and therefore, the system is asymptotic abelian, as a consequence of the usual locality condition. In statistical mechanics, however, one considers the time-translations. Therefore, the conditions of asymptotic abelianess is an extra requirement. Although one believes that timelike asymptotical abelianess is a consequence of the timelike decay of local excitations it never has been derived from the decay property. Nevertheless, timelike asymptotical abelianess has often been used in one form or other, as for example, in the proof of the equivalence of the K.M.S. with the stability condition [13]. We will treat these two situations separately. 


\section{A. Statistical Mechanics}

Here we are dealing with a $C^{*}$-dynamical system $\{\mathscr{A}, \mathbb{R}, \alpha\}$, and a temperature state $\omega_{\beta}$, which shall be a $\beta$-K.M.S. state. Looking at the G.N.S. representation $\{\pi(\mathscr{A}), U(t), \mathscr{H}, \Omega\}$ then as a consequence of the K.M.S. condition [12] the vector $\Omega$ is cyclic and separating for $\pi(\mathscr{A})$. In order to apply Theorem II.5 we remark that the two states $\omega_{0}$ and $\omega$ which are mentioned there, can be the same. We will assume that $\pi(\mathscr{A})$ is asymptotically abelian in a sense, which has to be made precise. Since the algebra $\pi(\mathscr{A})$ fulfills all the properties of the algebra $\mathscr{B}$ of Theorem II.5 we have to look for some bigger algebra $\mathscr{A}$ containing $\mathscr{B}$ such that $\mathscr{B}$ fulfills the quasi-duality condition. That this can be constructed is the content of the following:

IV.1 Proposition. Let $\{\mathscr{B}, \mathbb{R}, \beta\}$ be a $C^{*}$-dynamical system and let $\omega$ be an invariant state and $\{\pi, \mathscr{H}, \Omega\}$ be the G.N.S. triple defined by $\omega$. Assume that the vector $\Omega$ is cyclic and separating for $\pi(\mathscr{B})$ and assume $\pi(\mathscr{B})$ is $\pi$-strongly asymptotic abelian, which means

$$
\lim _{t \rightarrow \infty}\left\|\pi\left(A \beta_{t} B-\left(\beta_{t} B\right) A\right) \psi\right\|=0
$$

for every $\psi \in \mathscr{H}$. Then there exists a concrete $C^{*}$-dynamical system $\{\mathscr{A}, \mathbb{R}, \alpha\}$ acting on $\mathscr{H}$ such that

(i) $\mathscr{A}$ is weakly asymptotic abelian,

(ii) $\pi(\mathscr{B}) \subset \mathscr{A}$ and $\alpha_{t}$ restricted to $\pi(\mathscr{B})=\beta_{t}^{\pi}$.

Proof. Define $\beta_{t}^{\pi} \pi(A)=\pi\left(\beta_{t} A\right)$. By standard arguments we have a representation $U(t)$ of the group $\mathbb{R}$ implementing $\beta_{t}^{\pi}$, i.e. we have

$$
U(t) \pi(A) U^{*}(t)=\beta_{t}^{\pi} \pi(A) .
$$

This representation leaves the vector $\Omega$ invariant. Let $J$ be the modular conjugation for $\left\{\pi(\mathscr{B})^{\prime \prime}, \Omega\right\}$. Then $J$ commutes with $U(t)$. Define

$$
\mathscr{A}=C^{*}-\text { algebra generated by }\{\pi(\mathscr{B}) \cup J \pi(\mathscr{B}) J\} .
$$

and for $A \in \mathscr{A}$ define $\alpha_{t} A=U(t) A U^{*}(t)$. Since $\alpha_{t}$ maps $\pi(\mathscr{B})$ into itself and hence also $J \pi(\mathscr{B}) J$ into itself, it follows that $\alpha_{t}$ maps $\mathscr{A}$ into itself. We see from the construction that $\pi(\mathscr{B}) \subset \mathscr{A}$ and that $\alpha_{t}$ restricted to $\pi(\mathscr{B})=\beta_{t}^{\pi}$.

It remains to show that the algebra $\mathscr{A}$ is weakly asymptotic abelian. Let $1>$ $\varepsilon>0$ be given then for $A, B \in \mathscr{A}$ we can find $C_{i}^{\prime}, D_{i}^{\prime} \in \pi(\mathscr{B})$ and $C_{i}^{\prime}, D_{i}^{\prime} \in J \pi(\mathscr{B}) J$ with $\left\|\sum_{i=1}^{N} C_{i} C_{i}^{\prime}-A\right\| \leq \frac{\varepsilon}{12(\|B\|+1)}$ and with $\left\|\sum_{j=1}^{M} D_{i} D_{i}^{\prime}-B\right\| \leq \frac{\varepsilon}{12(\|A\|+1)}$. Using these estimates we obtain

$$
\begin{gathered}
\left\|[A, B]-\left[\sum C_{i} C_{i}^{\prime}, \sum D_{j} D_{j}^{\prime}\right]\right\| \leq 2\|B\|\left\|A-\sum C_{i} C_{i}^{\prime}\right\| \\
+2\left\|\sum C_{i} C_{i}^{\prime}\right\|\left\|B-\sum D_{j} D_{j}^{\prime}\right\| \leq \frac{\varepsilon}{3} .
\end{gathered}
$$

The same estimates hold if we replace $D_{j}$ and $D_{j}^{\prime}$ by $\alpha_{t} D_{j}$ and $\alpha_{j} D_{j}^{\prime}$. Next we get

$$
\sum_{i, j}\left(C_{i} C_{i}^{\prime} D_{j} D_{j}^{\prime}-D_{j} D_{j}^{\prime} C_{i} C_{i}^{\prime}\right)=\sum_{i, j} C_{i} D_{j}\left[C_{i}^{\prime}, D_{j}^{\prime}\right]+\left[C_{i}, D_{j}\right] D_{j}^{\prime} C_{i}^{\prime} .
$$


By strong abelianess of $\pi(\mathscr{B})$ and hence by that of $J \pi(\mathscr{B}) J$ we can find for every vector $\psi \in \mathscr{H}$ group elements $t_{i, j}$ and $t_{i, j}^{\prime}$ such that

$$
\left\|\left[C_{i}^{\prime}, \alpha_{t} D_{j}^{\prime}\right] \psi\right\| \leq \frac{\varepsilon}{3 N M\left\|C_{i} D_{j}\right\|}, \quad \text { for } \quad t>t_{i, j}^{\prime}
$$

and

$$
\left\|\left[C_{i}, \alpha_{t} D_{j}\right] \psi\right\| \leq \frac{\varepsilon}{3 N M\left\|D_{j}^{\prime} C_{i}^{\prime}\right\|}, \quad \text { for } t>t_{i, j}
$$

Putting now all terms together we obtain for $t>\max \left\{t_{i, j}, t_{i, j}^{\prime}\right\}$ the estimate

$$
\left|\left(\psi,\left[A, \alpha_{t} B\right] \psi\right)\right| \leq \varepsilon
$$

Using this result we obtain

IV.2 Theorem. Let $\left\{\mathscr{A}, \mathscr{A}(O), \mathbb{R}^{d}, \alpha\right\}$ be a theory of local observables and assume $\omega_{0}$ is an extremal $\beta$-K.M.S. state of $\mathscr{A}$ (with respect to the time translations). Denote by $\left\{\pi_{0}, \mathscr{H}_{0}, \Omega_{0}\right\}$ the G.N.S. triple defined by $\omega_{0}$. Assume $\pi_{0}(\mathscr{A})$ is $\alpha_{t}$ strongly asymptotically abelian in the sense of Eq. (11). If $V^{+}$denotes the forward light cone then one has

$$
S^{\prime}\left(\pi_{0}(\mathscr{A})^{\prime \prime}\right)=\operatorname{spec} \Delta_{\omega_{0}}^{\pi_{0}(\mathscr{A})^{\prime \prime}} \subseteq S^{\prime}\left(\pi_{0}\left(\mathscr{A}\left(V^{+}\right)\right)^{\prime \prime}\right) .
$$

If $\Omega_{0}$ is also cyclic for $\pi_{0}\left(\mathscr{A}\left(V^{+}\right)\right)$then the inclusion on the right-hand side is an equality.

Proof. In this situation $\omega_{0}$ will be identified with both of the states $\omega_{0}$ and $\omega$ mentioned in Theorem II.5. The forward light cone is clearly absorbing the whole of $\mathbb{R}^{d}$ for the time translation. If we denote the concrete $C^{*}$-algebra generated by two $C^{*}$-algebras $\mathscr{A}$ and $\mathscr{B}$ by $\mathscr{A} \vee \mathscr{B}$ then the triple

$$
\pi_{0}(\mathscr{A}) \vee J \pi_{0}(\mathscr{A}) J \supset \pi_{0}(\mathscr{A}) \supset \pi_{0}\left(\mathscr{A}\left(V^{+}\right)\right)
$$

fulfills the inclusion-, invariance-, quasi-duality-, and absorption requirements of Theorem II.5. Since our system is asymptotically abelian by Proposition IV.2 and since we have assumed that $\omega_{0}$ is an extremal K.M.S. state we conclude that $\omega_{0}$ fulfills the cluster property, see [18] and references therein. Hence we can use Theorem II.5 by taking for $\omega$ and $\omega_{0}$ the same state. This gives the statement of the theorem with the inclusion on the right-hand side. If $\Omega$ is cyclic for $\Pi\left(\mathscr{A}\left(V^{+}\right)\right)$ then we have the equality also on the right-hand side by the result of Araki [2] and Størmer [19].

Remarks. 1) From general investigation of K.M.S. states [12] it is known that the algebra $\pi(\mathscr{A})^{\prime \prime}$ is of type III. Hence this holds also for $\pi\left(\mathscr{A}\left(V^{+}\right)\right)^{\prime \prime}$.

2) In this theorem the forward light cone $V^{+}$can be replaced by any other set which is invariant under time translations in positive direction, and which absorbs $\mathbb{R}^{d}$.

3) If spacelike translations are also present in the considered representation then all the results we will describe under $B$. are correct in the present situation as long as we are staying in the same representation and the corresponding cyclicity assumptions are fulfilled. 


\section{B. Field Theory}

If we are dealing with the theory of local observables $\left\{\mathscr{A}, \mathscr{A}(O), \mathbb{R}^{d}, \alpha\right\}$ and if we take a spacelike direction $e$ and $\beta_{s}=\alpha_{s e}$ as group then the system is asymptotically abelian in the norm topology. In addition we have a large supply of pairs of subalgebras $\mathscr{A}\left(G_{1}\right) \supset \mathscr{A}\left(G_{2}\right)$ where the first one is $\beta$-invariant and the second one is absorbing the first.

Let us first describe the geometrical situation. Let $e$ be a spacelike direction then we will call a set $G \subset \mathbb{R}^{d} e$-semi-invariant, if for every $\lambda \in \mathbb{R}^{+}$one has $G+\lambda e \subset G$. It is called $e$-invariant if this relation holds for all $\lambda \in \mathbb{R}$. Having an $e$-semi-invariant domain $G$ then we obtain an invariant domain $G_{1}$ by

$$
G_{1}=\cup\{G+\lambda e ; \lambda \in \mathbb{R}\} .
$$

By this construction one has the situation that the domain $G$ is absorbing $G_{1}$, i.e. for every $a \in G_{1}$ exists $\lambda>0$ such that $a+\lambda e \in G$. Having a domain $G$, which is absorbing $G_{1}$ then in quantum field theory $\left\{\mathscr{A}, \mathscr{A}(O), \mathbb{R}^{d}, \alpha\right\}$ the corresponding algebras are also absorbing, which means the algebra $\mathscr{A}(G)$ absorbs $\mathscr{A}\left(G_{1}\right)$ with respect to the group $\alpha_{\lambda e}$. The most simple example of such absorbing pairs are spacelike cones $C$ which have the direction $e$ in their interior. In this case $C$ absorbs all of $\mathbb{R}^{d}$. If we, now, take a wedge $W$ such that $e$ lies in the intersection of the two defining lightlike hyperplanes, then $W \cap C$ absorbs $W$. One, now, can replace the cone $C$ by any other $e$-semi-invariant domain $G$ which absorbs $\mathbb{R}^{d}$. Also in this situation $W \cap G$ absorbs $W$.

In this construction the wedge $W$ is a set which is invariant by the shift $\lambda e$, $\lambda \in \mathbb{R}$. But one can replace this set by taking any other invariant set. This can be obtained from any set $O$ in the form

$$
Z=\bigcup_{\lambda \in \mathbb{R}}\{O+\lambda e\}
$$

We then again know that with any $e$-semi-invariant and $\mathbb{R}^{d}$ absorbing set $G$ the set $Z \cap G$ absorbs $Z$.

Before getting to the applications let us make a remark on the quasi-duality condition. If we are dealing with a theory of local observables which is also covariant under Lorentz-transformations and which in addition has the C.P.T.symmetry in the vacuum-sector then we have the duality-property for the wedge domain. This is a consequence of the work of Bisognano and Wichmann [3], which enables us to construct a field theory fulfilling the duality condition for a large family of domains. This discussion indicates that our much weaker quasiduality condition is fulfilled for a large class of field theories, as long as we are dealing with the vacuum sector.

After these preliminaries we first look at the vacuum-sector. We know by the work of Driessler [8] that in this situation the von Neumann-algebra of the wedge domain is of type $\mathrm{III}_{1}$, i.e. we have

$$
S^{\prime}\left(\pi_{0}(\mathscr{A}(W))^{\prime \prime}\right)=\mathbb{R}^{+} .
$$

With this we obtain:

IV.3 Theorem. Let $\left\{\mathscr{A}, \mathscr{A}(O), \mathbb{R}^{d}, \alpha\right\}$ be a theory of local observables and let $\left\{\pi_{0}, \mathscr{H}, \Omega_{0}\right\}$ be a vacuum representation with unique vacuum vector. Let $e$ be a spacelike direction, $G$ an e-semi-invariant domain absorbing all of $\mathbb{R}^{d}$ and $W$ an 
e-invariant wedge. If we assume that $\pi_{0}$ fulfills the quasi-duality condition then $\pi_{0}(\mathscr{A}(G \cap W))^{\prime \prime}$ is of type $I I I_{1}$.

Proof. Since we are dealing with a vacuum-representation we have by the ReehSchlieder-Theorem [17] that the vector $\Omega$ is cyclic and separating for the wedge $W$ as well as for $W \cap G$. Since we have assumed that $\pi_{0}(W)$ fulfills the quasi-duality condition we can apply Theorem III.2 and obtain

$$
S^{\prime}\left(\pi_{0}(\mathscr{A}(W))^{\prime \prime}\right)=S^{\prime}\left(\pi_{0}(\mathscr{A}(W \cap G))^{\prime \prime}\right) .
$$

Since by the result of Driessler the left-hand side is all of $\mathbb{R}^{+}$we obtain the stated result.

\section{Supplement}

( $\alpha$ ) If $\omega_{0}$ is only invariant, but, $\pi_{0}$ is not a vacuum representation and if $\Omega_{0}$ is cyclic and separating for the wedge and the spacelike cone $C$ then we know again from Driessler [9] that the von Neumann algebra $\pi(\mathscr{A}(W))^{\prime \prime}$ is of type III. But if we know that duality holds in addition, then the types of $\pi(\mathscr{A}(W \cap C))^{\prime \prime}$ are the same.

( $\beta$ ) In case we take a cylindrical set $Z$ instead of $W$ then we do not have much knowledge about the type of $\pi_{0}(\mathscr{A}(Z))^{\prime \prime}$ except $Z$ is generated by a double cone. In this case the algebra $\pi_{0}(\mathscr{A}(Z))^{\prime \prime}$ is of von Neumann type III [9]. If $\pi_{0}(\mathscr{A}(Z))$ fulfills the quasi-duality condition then we obtain by the same method

$$
S^{\prime}\left(\pi_{0}(\mathscr{A}(Z))^{\prime \prime}\right)=S^{\prime}\left(\pi_{0}(\mathscr{A}(Z \cap G))^{\prime \prime}\right),
$$

where $G$ is again any set which is $e$-semi-invariant and which absorbs $\mathbb{R}^{d}$.

Next we assume that $\omega$ and $\omega_{0}$ give rise to inequivalent representations. We will assume that $\omega$ and $\omega_{0}$ are asymptotically connected. What we have in mind are superselection sectors. So the G.N.S. triple $\{\pi, \mathscr{H}, \Omega\}$ represents a charged sector which is connected to the vacuum representation $\left\{\pi_{0}, \mathscr{H}_{0}, \Omega_{0}\right\}$ by large spacelike translations. If these superselection sectors are intertwined by localizable charged fields or by fields localized in spacelike cones then one can transport the knowledge about the types in the vacuum sector into the charged sector. If, however, the charged fields are not localizable then only our method gives results about the types of von Neumann-algebras in the charged sector. We obtain:

IV.4 Theorem. Let $\left\{\mathscr{A}, \mathscr{A}(O), \mathbb{R}^{d}, \alpha\right\}$ be a theory of local observables. Let e be a spacelike direction. Let $\omega_{0}, \omega$ be two states such that $\omega_{0}$ is a vacuum state and $\omega$ is asymptotically connected with $\omega_{0}$. Assume $G$ is the domain which is esemi-invariant and which absorbs $\mathbb{R}^{d}$. Let $W$ be an e-invariant wedge and assume $\pi_{0}(\mathscr{A}(W))$ fulfills the quasi-duality condition then $\pi(W \cap G)$ " is of type $I I I_{1}$.

Proof. Again by the Reeh-Schlieder-theorem [17] we know that $\Omega_{0}$ is cyclic and separating for $\pi_{0}(\mathscr{A}(W))$. Hence all conditions of Theorem III.2 are fulfilled and we obtain

$$
S^{\prime}\left(\pi(\mathscr{A}(W))^{\prime \prime}\right) \subset S^{\prime}\left(\pi(\mathscr{A}(W \cap G))^{\prime \prime}\right) .
$$

Since by the result of Driessler $S^{\prime}\left(\pi(\mathscr{A}(W))^{\prime \prime}\right)$ is all of $\mathbb{R}^{+}$, the same is true for the right-hand side. This implies $\pi(W \cap G)^{\prime \prime}$ is of type III $_{1}$. 


\section{Supplement}

$(\alpha)$ If the state $\omega_{0}$ is only an invariant state then we know that the von Neumann algebra belonging to the wedge regions is of type III, and hence $S^{\prime}$ contains the point 1. Then Theorem II.5 suffices to conclude that this is the same for the representation $\pi$. This does not necessarily mean that these algebras have the same Connes-von Neumann type, but the von Neumann type must be the same. Again this holds under the assumption of quasi-duality in the representation $\pi_{0}$. $(\beta)$ In case we take a cylindrical set $Z$ instead of $W$ then we do not have much knowledge about the type of $\pi_{0}(Z)^{\prime \prime}$. If $\pi_{0}(Z)$ fulfills the quasi-duality condition then we obtain by the same method

$$
S^{\prime}\left(\pi_{0}(\mathscr{A}(Z))^{\prime \prime}\right) \subseteq S^{\prime}\left(\pi(\mathscr{A}(Z \cap G))^{\prime \prime}\right),
$$

where $G$ is again any set which is $e$-semi-invariant and which absorbs $\mathbb{R}^{d}$.

Remarks. (i) Our discussion is by far not exhaustive. If, for instance, one can prove the scaling property only in the vacuum sector, then one can transport by our method the knowledge about types into other superselection sectors. We obtain that $\pi(\mathscr{C})^{\prime \prime}$ is of type $\mathrm{III}_{1}$ for a large family of domains.

(ii) This method is not restricted to the translation group alone. Other groups, as Lorentz-boosts, dilatations, and etc. can serve the same purpose, provided the corresponding assumptions are made. We have restricted ourselves to large spacelike translations, because the assumptions used here are natural in the theory of superselection sectors.

(iii) In the case where one is using scaling limits the method could be transported to curved space-time manifolds (see e.g. Wollenberg [20]). This is also true in our situation, provided the manifold has so much symmetry that an appropriate group is present.

(iv) Starting from Wightman-fields, instead of rings of bounded operators, is another possibility. But we refrain from discussing it because the questions connected with the passage from fields to local algebras is not yet completely solved. For a review on this transition problem see e.g. Yngvason [21].

Acknowledgement. The second named author would like to thank the Institut für Theoretische Physik, Universität Göttingen, for the kind hospitality and financial support.

\section{References}

1. Araki, H.: Type of von Neumann algebras associated to the free field. Progr. Theor. Phys. 32, 956-961 (1964)

2. Araki, H.: Remarks on the spectra of modular operators of von Neumann algebras. Commun. Math. Phys. 28, 267-277 (1972)

3. Bisognano, J., Wichmann, E.H.: On the duality for a hermitian scalar field. J. Math. Phys. 16, 985-1007 (1975)

4. Borchers, H.-J.: Local rings and the connection of spin with statistics. Commun. Math. Phys. 1, 281-307 (1965)

5. Buchholz, D., Fredenhagen, K.: Locality and the structure of particle states. Commun. Math. Phys. 84, 1-54 (1982)

6. Buchholz, D., D'Antoni, C., Fredenhagen, K.: The universal structure of local algebras. Commun. Math. Phys. 11, 123-135 (1987)

7. Connes, A.: Un nouvel invariant pour le algebra de von Neumann. Compl. Rend. Acad. Sci. Paris, Ser. A 273, 900-903 (1971) 
8. Driessler, W.: Comments on lightlike translations and applications to relativistic quantum field theory. Commun. Math. Phys. 44, 133-141 (1975)

9. Driessler, W.: On the type of local algebras in quantum field theory. Commun. Math. Phys. 53, 295-297 (1977)

10. Doplicher, S., Haag, R., Roberts, J.E.: Local observables and particle statistics. Commun. Math. Phys. 23, 199-230 (1971); Commun. Math. Phys. 35, 49-85 (1974)

11. Fredenhagen, K.: On the modular structure of local algebras of observables. Commun. Math. Phys. 97, 79-89 (1985)

12. Haag, R., Hugenholtz, N.M., Winnik, M.: On the equilibrium state in quantum statistical mechanics. Commun. Math. Phys. 5, 215-236 (1967)

13. Haag, R., Kastler, D., Trych-Pohlmeyer, E.B.: Stability and equilibrium states. Commun. Math. Phys. 38, 173-193 (1974)

14. Haag, R., Narnhofer, H., Stein, U.: On quantum field theory in gravitational background. Commun. Math. Phys. 94, 219-238 (1984)

15. Hislop, P.D., Longo, R.: Modular structure of the local algebra asociated with a free massless scalar field theory. Commun. Math. Phys. 84, 71-85 (1982)

16. Longo, R.: Algebraic and modular structure of von Neumann algebras in physics. Proc. Symp. Pure Math. 38, 551-566 (1982)

17. Reeh, H., Schlieder, S.: Bemerkungen zur Unitäräquivalenz von Lorentzinvarianten Feldern. Nuovo Cimento 22, 1051-1068 (1961)

18. Størmer, E.: Large groups of automorphisms of $C^{*}$-algebras. Commun. Math. Phys. 5, 1-22 (1967)

19. Størmer, E.: Spectra of states and asymptotically abelian $C^{*}$-algebras. Commun. Math. Phys. 28, 279-294 (1972); Commun. Math. Phys. 38, 341-343 (1974)

20. Wollenberg, M.: Scaling limits and type of local algebras over curved space-time. Preprint, Berlin 1989

21. Yngvason, J.: Bounded and unbounded realizations of locality. In: Proceedings of the IXth IAMP Congress, Swansea 1988. Simon, B., Davies, I.M., Truman, A. (eds.) Bristol: Hilger 1989 
